\title{
Is fluorescein-guided technique able to help in resection of high-grade gliomas?
}

\author{
Francesco Acerbi, M.D., Ph.D., ${ }^{1}$ Morgan Broggi, M.D., ${ }^{1}$ Marica Eoli, M.D., ${ }^{2}$ \\ Elena Anghileri, M.D., Ph.D., ${ }^{2}$ Claudio Cavallo, M.S., ${ }^{1}$ Carlo Boffano, M.D., 5 \\ Roberto Cordella, Ph.D., ${ }^{1}$ Lucia Cuppini, Ph.D., ${ }^{2}$ Bianca Pollo, M.D., ${ }^{3}$ \\ Marco Schiariti, M.D., ${ }^{1}$ Sergio Visintini, M.D., ${ }^{1}$ Chiara Orsi, ${ }^{4}$ \\ Emanuele La Corte, M.S., ${ }^{1}$ Giovanni Broggi, M.D., ${ }^{1}$ and Paolo Ferroli, M.D. ${ }^{1}$
}

Departments of ${ }^{I}$ Neurosurgery, ${ }^{2}$ Molecular Neuro-Oncology, ${ }^{3}$ Neuropathology, and ${ }^{5}$ Neuroradiology, Fondazione IRCCS Istituto Neurologico Carlo Besta, Milano; and ${ }^{4}$ Department of Biostatistics and Clinical Epidemiology, Department of Public Health, Forensic and Experimental Medicine, University of Pavia, Italy

\begin{abstract}
Object. Fluorescein, a dye that is widely used as a fluorescent tracer, accumulates in cerebral areas where the blood-brain barrier is damaged. This quality makes it an ideal dye for the intraoperative visualization of high-grade gliomas (HGGs). The authors report their experience with a new fluorescein-guided technique for the resection of HGGs using a dedicated filter on the surgical microscope.

Methods. The authors initiated a prospective Phase II trial (FLUOGLIO) in September 2011 with the objective of evaluating the safety of fluorescein-guided surgery for HGGs and obtaining preliminary evidence regarding its efficacy for this purpose. To be eligible for participation in the study, a patient had to have suspected HGG amenable to complete resection of the contrast-enhancing area. The present report is based on the analysis of the short- and long-term results in 20 consecutive patients with HGGs (age range 45-74 years), enrolled in the study since September 2011.

In all cases fluorescein $(5-10 \mathrm{mg} / \mathrm{kg}$ ) was injected intravenously after intubation. Tumor resection was performed with microsurgical technique and fluorescence visualization by means of BLUE 400 or YELLOW 560 filters on a Pentero microscope.

Results. The median preoperative tumor volume was $30.3 \mathrm{~cm}^{3}$ (range 2.4-87.8 $\mathrm{cm}^{3}$ ). There were no adverse reactions related to fluorescein administration. Complete removal of contrast-enhanced tumor was achieved in $80 \%$ of the patients. The median duration of follow-up was 10 months. The 6-months progression-free survival rate was $71.4 \%$ and the median survival was 11 months.

Conclusions. Analysis of these 20 cases suggested that fluorescein-guided technique with a dedicated filter on the surgical microscope is safe and allows a high rate of complete resection of contrast-enhanced tumor as determined on early postoperative MRI. Clinical trial registration no.: 2011-002527-18 (EudraCT).

(http://thejns.org/doi/abs/10.3171/2013.11.FOCUS13487)
\end{abstract}

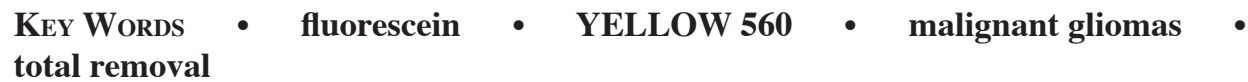

$\mathrm{H}$ IGH-GRADE gliomas (HGGs) are associated with a poor prognosis despite maximal treatment with combination therapy involving surgery, chemotherapy, and radiotherapy. ${ }^{26}$ Some retrospective studies and one prospective study have suggested a significant effect of extent of resection on survival of patients with these aggressive tumors. ${ }^{7,11,13,21}$ It is, however, difficult to distinguish viable tumor tissue at the margins of resection during surgery, ${ }^{29}$ and complete removal, defined as surgical excision of the entire enhancing mass, is therefore

\footnotetext{
Abbreviations used in this paper: $\mathrm{AA}=$ anaplastic astrocytoma; $\mathrm{GBM}=$ glioblastoma; $\mathrm{HGG}=$ high-grade glioma; iMRI = intraoperative MRI; KPS = Karnofsky Performance Status; MGMT = O-6-methylguanine-DNA methyltransferase; MMSE = Mini Mental State Evaluation; NIHSS = National Institutes of Health Stroke Scale; OS = overall survival; $\mathrm{PFS}=$ progression-free survival; 5-ALA = 5-aminolevulinic acid.
}

difficult to achieve. Studies have shown it to be feasible in only a small proportion of cases.. ${ }^{11,13}$ Technical tools, such as neuronavigation, ${ }^{32}$ intraoperative MRI (iMRI), ${ }^{6,15}$ and ultrasonography, ${ }^{30}$ have been applied in an effort to improve surgical results, but only iMRI has been studied with specific application to glioma surgery in a randomized trial. The results of this trial provided Class I evidence for the effect of this technique on extent of resection. ${ }^{19}$ However, no difference in progression-free survival (PFS) was found between the two groups included in this study. ${ }^{19}$ In addition, iMRI-guided surgery can take significantly more time, and can interfere with the surgical workflow (for example, because of issues regarding compatibility of instruments or equipment) depending on the sort of iMRI technology used. ${ }^{6}$ Photodynamic detection of the tumor, which takes advantage of a photosensitive drug specific for the tumor tissue and with a well-defined fluorescence, has been used in neurosurgery during removal 
of HGGs. In particular, the utilization of 5-aminolevulinic acid (5-ALA), a natural biochemical precursor of hemoglobin that evokes synthesis and accumulation of fluorescent porphyrins in some kinds of tumors, including gliomas, has been shown to be useful during surgical removal of HGGs, ${ }^{22,24,25}$ and it has been shown to increase the rates of complete resection and 6-month PFS in a randomized trial. ${ }^{23}$ However, some limitations were found with this technique: the drug should be administered orally 2.5 to 3.5 hours before induction of anesthesia; it is recommended not to expose the patient to sunlight or strong room light for 24 hours after administration because of the risk of skin sensitization; ${ }^{29}$ and 5-ALA is expensive (approximately 900 Euros per vial).

Fluorescein sodium, the sodium salt of fluorescein, provides an attractive alternative for fluorescence-guided resection of HGGs. It is far less expensive than 5-ALA (approximately 5 Euros per 1-g vial) and has been extensively used intravenously as a diagnostic tool to perform retinal angiography, with a very low rate of side effects.9,10,16,33 Due to its ability to penetrate areas of the brain where the blood-brain barrier has been disrupted, it has been used with the purpose of localizing brain tumors during open biopsy since $1948 .{ }^{14}$ In addition, the feasibility of fluorescein-guided removal of HGGs has been suggested by the results of several studies. ${ }^{8,18,20}$ The results of one prospective study suggested a possible improvement of the extent of resection using fluorescein, although in that study surgery was performed without a specific filter integrated into the surgical microscope. ${ }^{5}$ In 2011, our group started the first prospective Phase-II trial with the aid of a microscope-integrated fluorescence kit (FLUOGLIO) to evaluate the safety of and obtain initial data about the efficacy of fluorescence-guided resection of HGGs. The study was based on Simon's two-stage design. In 2013, we reported the preliminary short-term results in 12 cases of glioblastoma (GBM). ${ }^{1}$

In the present paper we analyzed the short and longterm results of fluorescein-guided resection using a dedicated filter on the surgical microscope in the first 20 patients with HGGs enrolled in the FLUOGLIO study.

\section{Methods}

\section{Patients}

The inclusion criteria of the FLUOGLIO trial were as follows: 1) age 18 to 75 years; 2) suspected, newly diagnosed, untreated HGGs (with the tentative diagnosis being based on brain MRI with and without contrast and with volumetric sequences); 3) tumor location allowing for complete resection of contrast-enhancing area as determined by the surgeon. The exclusion criteria were: 1) a histological diagnosis other than HGG (anaplastic astrocytoma [AA] or GBM); 2) a tumor that originated in the midline, basal ganglia, cerebellum, or brainstem; 3) multicentric tumors; 4) the presence of a large, non-contrastenhancing area, suggesting low-grade glioma with malignant transformation; 5) medical reasons precluding MRI (for example, the presence of a pacemaker); 6) inability to give consent because of dysphasia or language barrier;
7) Karnofsky Performance Status (KPS) score of 60 or less; 8) renal insufficiency; 9) hepatic insufficiency; and 10) history of active malignant tumors at any other site.

The study started in September 2011, when the first patient was enrolled. Written informed consent was obtained from all patients. The FLUOGLIO study was approved by the Ethics Committee of the Foundation IRCCS Neurological Institute Carlo Besta of Milan and registered on the European Regulatory Authorities website (EudraCT no. 2011-002527-18).

Twenty-two patients (16 male and 6 female) were screened for participation in this prospective trial. The patients' age ranged from 45 to 74 years (median 66 years). Two patients were excluded due to histological diagnoses other than HGG (lymphoma in 1 case and primitive neuroectodermal tumor in the other). Twenty patients were finally enrolled (19 with GBM and 1 with AA).

Clinical and neurological characteristics of the patients are summarized in Table 1.

\section{Clinical and Radiological Assessment}

The KPS was used to record the patients' general physical status both pre- and postoperatively. Data from laboratory tests were also recorded. The patients' neurological function was assessed by means of the Mini Mental State Evaluation (MMSE) and the National Institutes of Health Stroke Scale (NIHSS). Clinical assessment consisted of a preoperative visit within 14 days of surgery and an immediate postoperative evaluation within the first 72 hours after surgery.

All patients underwent MRI studies with a 1.5-T Siemens scanner with and without the use of a contrast agent. In all cases volumetric sequences were included. The preoperative MRI studies were performed within 4 days of surgery, and the postoperative studies within the first 72 hours after surgery. The preoperative tumor volume and the postoperative residual tumor volume were calculated by an independent neuroradiologist (C.B.), using open-source software (Osirix for Macintosh, www. osirix-viewer.com). Residual tumor was defined as an area of contrast-enhancement with a calculated volume greater than $0.175 \mathrm{~cm}^{3} .{ }^{23}$

After surgery, all patients were treated according to the Stupp protocol with radiotherapy and concomitant temozolomide therapy. ${ }^{27}$ No restrictions were imposed on treatment after disease progression. Any further treatment after the Stupp protocol (that is, second-line chemotherapy, additional surgery and radiosurgery) was recorded.

Patients underwent clinical and radiological followup examinations within 4 weeks after the end of radiochemotherapy and then every 2 months (for GBM patients) or every 3 months (for the patient with AA). The clinical evaluation included the same tests performed at the preoperative and early postoperative evaluations. Imaging follow-up included MRI with and without contrast agent, including volumetric sequences (Siemens 1.5 T).

We defined progression at follow-up MRI as the appearance of a new contrast-enhancing area greater than $0.175 \mathrm{~cm}^{3}$ or an increase in residual tumor based on the RANO criteria. $^{31}$ 
Fluorescein-guided removal of high-grade gliomas

TABLE 1: Clinical characteristics of the patients included in FLUOGLIO study from September 2011 to June 2013

\begin{tabular}{|c|c|c|c|c|c|c|c|c|c|}
\hline \multirow[b]{2}{*}{ Case No. } & \multirow[b]{2}{*}{ Tumor Location } & \multirow[b]{2}{*}{ Tumor Size $\left(\mathrm{cm}^{3}\right)$} & \multirow[b]{2}{*}{ Histology } & \multirow[b]{2}{*}{ MGMT Status } & \multirow[b]{2}{*}{$\%$ of Resection } & \multicolumn{2}{|c|}{ NIHSS } & \multicolumn{2}{|c|}{ KPS Score } \\
\hline & & & & & & Preop & Postop & Preop & Postop \\
\hline 1 & It occipital & 28.0 & GBM & unmethylated & 100 & 3 & 4 & 70 & 60 \\
\hline 2 & It temporal & 12.8 & GBM & methylated & 100 & 0 & 0 & 100 & 100 \\
\hline 3 & rt parietal & 49.8 & GBM & unmethylated & 100 & 3 & 4 & 70 & 80 \\
\hline 4 & rt parietal & 87.8 & GBM & methylated & 99.9 & 7 & 8 & 70 & 60 \\
\hline 5 & rt frontal & 12.2 & GBM & unmethylated & 100 & 0 & 1 & 90 & 80 \\
\hline 6 & It occipital & 41.0 & GBM & unmethylated & 100 & 0 & 0 & 100 & 100 \\
\hline 7 & It temporoinsular & 31.8 & GBM & unmethylated & 88.6 & 1 & 2 & 90 & 80 \\
\hline 8 & It frontoinsular & 34.5 & GBM & unmethylated & 100 & 4 & 13 & 90 & 50 \\
\hline 9 & rt frontal & 9.6 & GBM & unmethylated & 82.8 & 0 & 3 & 90 & 70 \\
\hline 10 & It temporal & 2.4 & $\mathrm{AA}$ & methylated & 100 & 1 & 1 & 90 & 90 \\
\hline 11 & rt frontal & 20.0 & GBM & unmethylated & 100 & 1 & 1 & 90 & 90 \\
\hline 12 & rt frontal & 83.8 & GBM & unmethylated & 100 & 2 & 1 & 80 & 90 \\
\hline 13 & rt temporal & 86.1 & GBM & unmethylated & 100 & 1 & 0 & 70 & 80 \\
\hline 14 & rt parietal & 12.5 & GBM & unmethylated & 100 & 1 & 4 & 100 & 90 \\
\hline 15 & rt frontal & 69.7 & GBM & unmethylated & 100 & 2 & 5 & 70 & 50 \\
\hline 16 & It frontal & 8.3 & GBM & unmethylated & 100 & 1 & 2 & 90 & 70 \\
\hline 17 & rt frontal & 31.8 & GBM & methylated & 100 & 0 & 0 & 100 & 100 \\
\hline 18 & rt frontal & 8.9 & GBM & unmethylated & 100 & 0 & 0 & 90 & 90 \\
\hline 19 & It temporal & 28.8 & GBM & unmethylated & 100 & 1 & 2 & 80 & 70 \\
\hline 20 & rt parietal & 38.9 & GBM & unmethylated & 99.3 & 1 & 2 & 90 & 80 \\
\hline
\end{tabular}

Because general anesthesia was used for surgery, immediate and postoperative reactions to the administration of fluorescein could be evaluated only for objective findings, such as hypotension, cardiac arrest, shock, seizures, bronchospasm, or anaphylaxis.

\section{Surgical Protocol}

Our surgical protocol of fluorescein-guided technique has been described in a previous report of the preliminary results of the FLUOGLIO trial. ${ }^{1}$

Briefly, after intubation and before skin incision, patients received $5-10 \mathrm{mg} / \mathrm{kg}$ of a $20 \%$ solution of sodium fluorescein (Monico Spa, Italy), administered intravenously. A dedicated surgical microscope (Pentero with fluorescence kit, BLU 400 or YELLOW 560, Carl Zeiss) was used during the surgical procedure. For the first 6 cases, we used the same fluorescence kit as for 5-ALAguided glioma resection (BLU 400). A new fluorescence kit, YELLOW 560, became available in January 2012 and was used in subsequent cases. This kit was specifically designed by the Carl Zeiss Company for excitation in the wavelength range of 460-500 $\mathrm{nm}$ and for observation in the wavelength range of 540-690 $\mathrm{nm}$. Consequently, it was possible to reduce the fluorescein dosage to $5 \mathrm{mg} / \mathrm{kg}$. Neuronavigation was allowed only for surgical planning, initial tumor localization, and orientation during tumor removal, but not for judgment regarding extent of resection. Ultrasonography was not used. For tumors located in or close to eloquent areas, neurophysiological monitoring was performed following our usual protocol. ${ }^{2}$ During resection, the microscope could be switched alternatively from fluorescent to white-light illumination, as desired by the surgeon, by pressing the specific button on the microscope handgrip. However, with the YELLOW 560 filter, as already described, it was possible to visualize the fluorescent signal and at the same time the nonfluorescent tissue in more natural color. ${ }^{1}$ For this reason, most of the surgery was completed with the filter activated. Tumor resection was completed in an inside-out fashion, usually by ultrasonic aspiration, until all the fluorescent tissue exposed was removed, as considered feasible by the surgeon (Figs. 1 and 2).

\section{Histological Analysis and MGMT Methylation Status}

Histological analysis of the tumors was performed in a standard manner and tumors were classified according to the 2007 WHO classification by an independent neuropathologist (B.P.). ${ }^{12,17}$ For patients who had tumors in noneloquent areas and gave their specific written informed consent, biopsies were performed at the tumor margin to evaluate the accuracy of fluorescein in identification of tumor tissue. Four biopsy specimens were obtained at the tumor margin in areas located distant from each other-2 in the fluorescent tissue and 2 in the nonfluorescent tissue-to calculate sensitivity and specificity (as described in Statistical Analysis, below). In these cases, histological analysis was performed by a pathologist who was blind to the fluorescence characteristics of the biopsies, to discriminate between clear tumor tissue and peritumor area (gliosis or tumor cell infiltration), also performing immunohistochemistry for GFAP (glial fibrillary acidic protein) and for the proliferation index with $\mathrm{Ki} 67$ (MIB 1). 

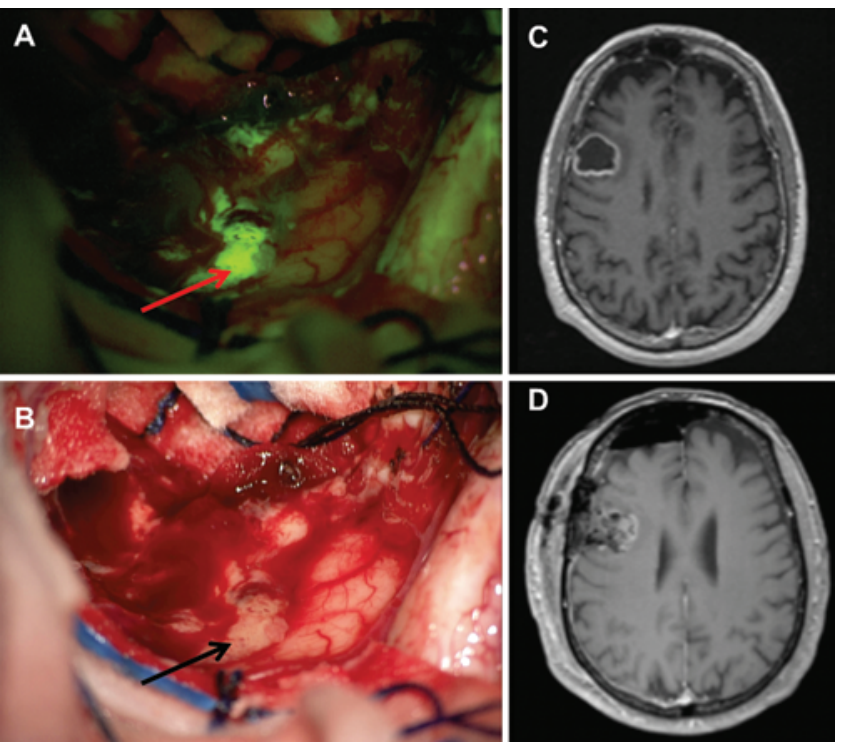

FIG. 1. Case 18. A and B: Intraoperative view during resection of a right frontal GBM. With the YELLOW 560 filter activated (A) it is possible to visualize an area of tumor that needs to be removed (red arrow); the same view is shown with white light illumination (B), with the tumor tissue indicated by a black arrow. Note in A (right part of the picture) the reflected dural flap appearing fluorescent (see text) C: Preoperative axial T1-weighted MR image obtained after intravenous administration of a contrast agent showing a right frontal GBM (tumor volume $8.93 \mathrm{~cm}^{3}$ ). D: Early postoperative (< 48 hours after surgery) axial T1weighted MR image obtained after contrast administration showing a complete tumor resection without any area of contrast enhancement.

Methylation patterns in the $\mathrm{CpG}$ islands of O-6-methylguanine-DNA methyltransferase (MGMT) were also analyzed. ${ }^{4}$

\section{Statistical Analysis}

The sample size and stopping rules of the FLUOGLIO trial have been described in a previous report. ${ }^{1}$

The sample was described by means of the usual descriptive statistics: mean, median, and standard deviation for continuous variables and proportions for categorical ones. The Student t-test was used to compare pre- and postoperative clinical status.

Sensitivity and specificity were calculated to evaluate fluorescein accuracy in tumor identification as follows. Sensitivity was calculated as the number of true-positive fluorescent samples (histologically confirmed HGG)/all samples with confirmed HGG. Specificity was calculated as the number of true-negative nonfluorescent samples (histologically confirmed as not neoplastic)/all samples histologically confirmed as not neoplastic.

Progression-free survival (PFS) was calculated from the time of surgery until disease progression or death/last follow-up, if censored. Six-month PFS was defined as the proportion of patients without radiological progression at the 6th month after surgery. Overall survival (OS) was calculated from treatment onset until death or last followup, if censored; PFS and OS were estimated by the Kaplan-Meier method.
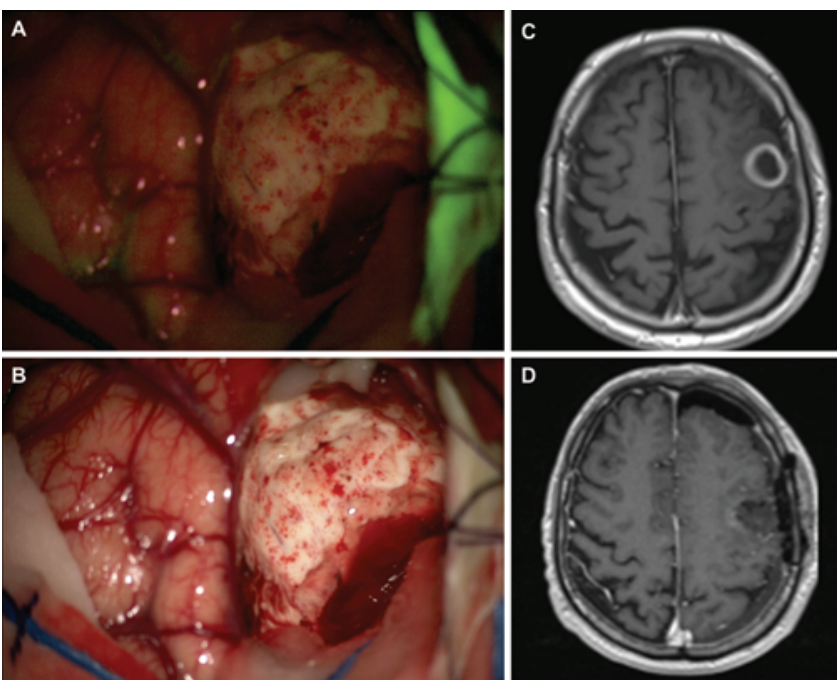

FIG. 2. Case 16. A and B: Intraoperative view during resection of a left frontal GBM. At the end of the resection with the YELLOW 560 filter activated $(A)$ there is no more fluorescent area visible; the same view is also shown with white light illumination (B). Note in $A$ (right part of the picture) that the reflected dural flap is still fluorescent after tumor removal (see text). C: Preoperative axial T1-weighted MR image obtained after contrast administration showing a left frontal GBM (tumor volume $8.26 \mathrm{~cm}^{3}$ ). D: Early postoperative (< 48 hours after surgery) contrast-enhanced axial T1-weighted MR image showing a complete tumor resection without any area of contrast enhancement.

\section{Results}

\section{Intraoperative Fluorescence Characteristics}

The mechanism of action of fluorescein is related to the passage through a damaged blood-brain barrier and accumulation in extracellular space. Thus, it helps in visualizing the pathological tissue in the same way as contrast enhancement during MRI.

We injected fluorescein after patient intubation and before skin incision. In this way, we believed that we could avoid accumulation of fluorescein in cerebral areas inadvertently damaged during craniotomy or dural opening. The microscope is brought into the operative field after craniotomy. At the activation of the filter, the dura appeared fluorescent due to the characteristic features of its vascularization (Figs. 1 and 2). Tumor tissue showed an intense fluorescence, even if some necrotic parts of tumors appeared darker. Sometimes, in cases of partly cystic lesions, the liquid inside the cyst was intensively fluorescent and leaked during resection until full aspiration.

Regarding the 2 filters, as already described in our preliminary experience, both the BLU 400 and YELLOW 560 modules of the Zeiss microscope allowed a good discrimination between the fluorescent and nonfluorescent tissue. ${ }^{1}$ In fact, due to the wide range of emission and excitation, even the BLU 400 filter, which was used in the first 6 cases, allowed a clear visualization of the light emitted by fluorescein in the area of blood-brain barrier disruption. The main difference between the two filters was related to the fact that, due to the higher specificity of YELLOW 560 module, the fluorescent tissue appeared greener and brighter, with a dose of fluorescein that was 
reduced by half. In addition, due to the specific YELLOW 560 module characteristics, ${ }^{1}$ the nonfluorescent tissue appeared in more natural color, without significant differences between the oculars of the first surgeon and those of the assistant (Fig. 1). This reduced eye fatigue during surgery. Furthermore, in this way, most of the procedure could be performed under filter activation, reducing the discomfort of switching continuously between white light and fluorescence illumination to control bleeding or manipulate vessels during tumor resection.

\section{Percentage of Resection}

The median preoperative tumor volume was $30.3 \mathrm{~cm}^{3}$ (range 2.4-87.8 $\mathrm{cm}^{3}$ ). Complete removal of all enhancing tumor as verified by an early postoperative MRI was achieved in 16 cases (80\%) (Fig. 3 and Table 1). In the remaining cases the mean extent of tumor resection was 92.6\% (range 82.8\%-99.9\%) (Fig. 4 and Table 1).

\section{Accuracy of Fluorescein in Tumor Identification}

A total of 36 biopsies (18 in fluorescent tissue and 18 in nonfluorescent tissue) were performed at the tumor margin in 9 patients (4 biopsies in each patient). Sixteen of the 18 biopsies of fluorescent areas showed clear HGG tissue. Seventeen of the 18 biopsies of nonfluorescent areas showed the absence of HGG tissue. These data were confirmed by immunohistochemical analysis, as previously reported. ${ }^{1}$ Therefore, we obtained a sensitivity and a specificity of fluorescein in identifying tumor tissue, respectively, of $94 \%$ and $89.5 \%$.

\section{Reactions to Fluorescein, Postoperative Neurological Status, and Complications}

No adverse reaction to fluorescein administration was registered in this cohort of patients, including those with different histological diagnoses who were excluded from the analysis, although, as described above, only objective findings could be recorded due to the fact that patients were in a state of general anesthesia during surgery.

No statistically significant difference was found between preoperative and immediate postoperative NIHSS scores $(1.45 \pm 0.38$ vs $2.65 \pm 0.71, \mathrm{p}=0.148)$ and KPS scores (median 90 vs $80, \mathrm{p}=0.145$ ).

In the early postoperative period (during the first postoperative month), complications were observed in 3 cases. One patient with left occipital GBM (Case 1) developed an entrapped temporal horn 16 days after surgery and underwent ventriculoperitoneal shunt insertion. One patient with left frontoinsular GBM (Case 8) developed an infarction in the internal capsule causing hemiplegia and aphasia; the patient partially recovered after intensive postoperative rehabilitation. One patient with right frontal GBM (Case 18) had a postoperative infection of the bone flap, requiring wound revision and antibiotic therapy.

\section{Progression-Free and Overall Survival}

Fifteen of 20 patients completed the Stupp protocol postoperatively. After a median follow-up of 10 months (range 3-25 months), the estimated 6-month PFS was $71.4 \%(60.6 \%-82.2 \%)$ (Fig. 5). Eleven patients died dur-
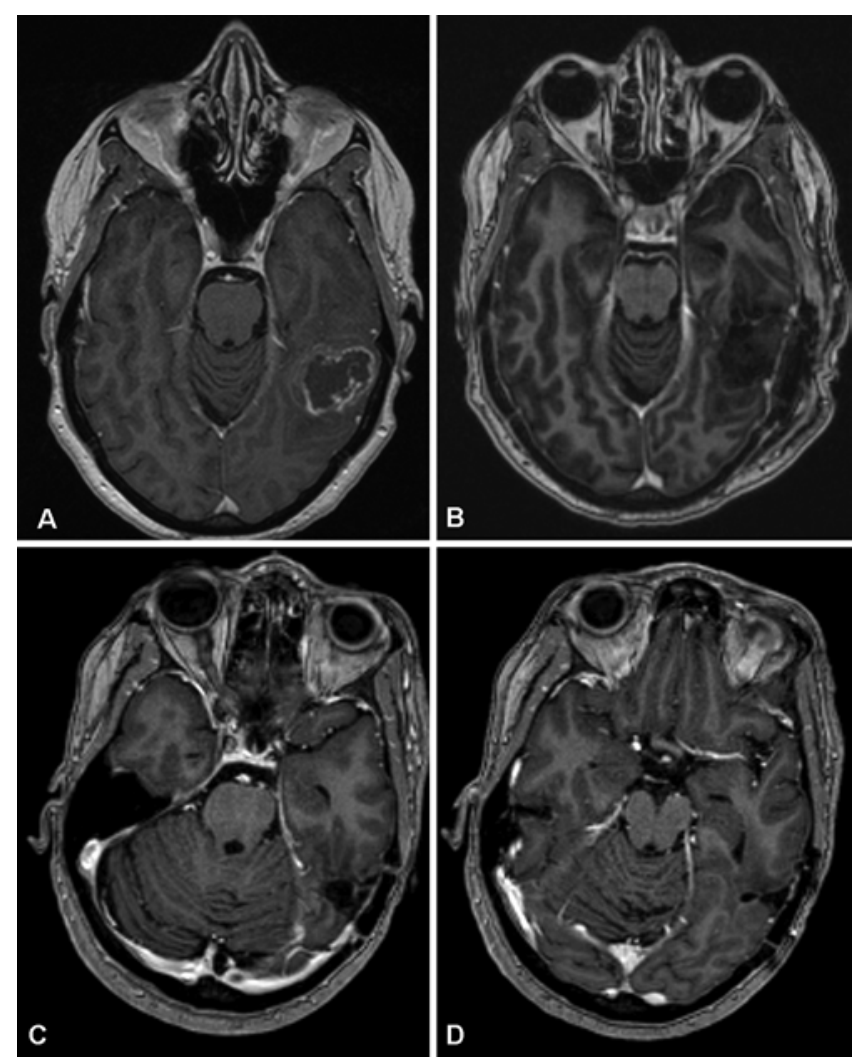

FIG. 3. Case 2. A: Preoperative axial T1-weighted MR obtained after contrast administration showing a left posterior temporal GBM (tumor volume $\left.12.8 \mathrm{~cm}^{3}\right)$. B: Early postoperative $(<48$ hours after surgery) axial T1-weighted MR image obtained after contrast administration showing a complete tumor resection without any area of contrast enhancement. C and D: Follow-up contrast-enhanced axial T1-weighted MR image obtained 24 months after surgery showing no tumor recurrence.

ing the follow-up period as a result of tumor progression (10 cases) or an unrelated cause (myocardial infarction in 1 case, Case 15). The estimated median survival was 11 months (Fig. 6).

\section{Discussion}

Our data in this cohort of patients confirmed that the use of intravenous fluorescein during resection of HGGs is safe and showed that it was associated with a high rate of complete resection of contrast-enhanced tumor at the early postoperative MRI.

As already suggested by our preliminary analysis of a subgroup of patients with $\mathrm{GBM}^{1}$ and based on the known safety profile of fluorescein, no adverse reaction related to the use of this agent has been recorded so far in the FLUOGLIO study. This might be related also to the use of specific filters that enable reduction of the total dose of fluorescein injected ${ }^{1,8,18}$ with a possible impact on reduction of side effects. Rare adverse events, including anaphylactic reactions, after fluorescein injection have been reported in the ophthalmological and neurosurgical literature, , $^{3,10,16,28,33}$ but no side effects were registered with the use of low-dose fluorescein in more recent pa- 

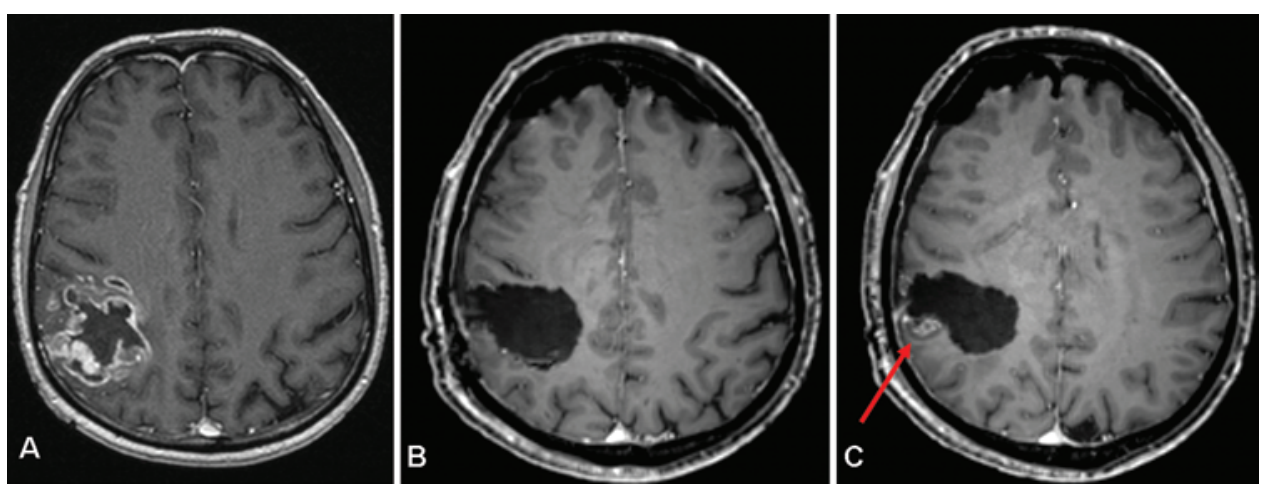

FIG. 4. Case 20. A: Preoperative axial T1-weighted MR image obtained after contrast administration showing a right frontal GBM (tumor volume $\left.38.92 \mathrm{~cm}^{3}\right)$. B and C: Early postoperative (< 48 hours after surgery) axial T1-weighted MR image obtained after contrast administration showing a very extensive, but not complete tumor removal (red arrow in $\mathrm{C}$ ); the residual tumor volume was $0.28 \mathrm{~cm}^{3}$.

pers. In addition, it should be noticed that in neurosurgical applications, side effects may be even less frequent than in ophthalmological cases due to the fact that fluorescein is administered with the patient intubated in a state of general anesthesia.

The volumetric analysis performed in our cohort of 20 patients with HGGs on early postoperative MRI showed a total resection of the contrast-enhancing tissue in $80 \%$ of cases. These data confirmed the results of our previous preliminary analysis of 12 cases. ${ }^{1}$ Although still limited by the small number of cases and, more importantly, the absence of a control group of patients undergoing surgery without fluorescein, the results were promising. The inclusion and exclusion criteria were in fact similar to those of the prospective Phase III trial on 5-ALA by Stummer et al. ${ }^{23}$ Specifically, we enrolled patients who had tumors in locations that allowed for complete resection of the contrast-enhanced area. In a control group of patients from the 5-ALA study, operated on without the aid of fluorescent visualization, a complete resection was reported to be feasible only in $36 \%$ of the cases. ${ }^{23}$ However, it should be emphasized that the accumulation of fluorescein in the brain tumor area is related to the passage through a damaged blood-brain barrier, and not to a specific uptake by the high-grade glial cells, as for 5-ALA. Therefore, this

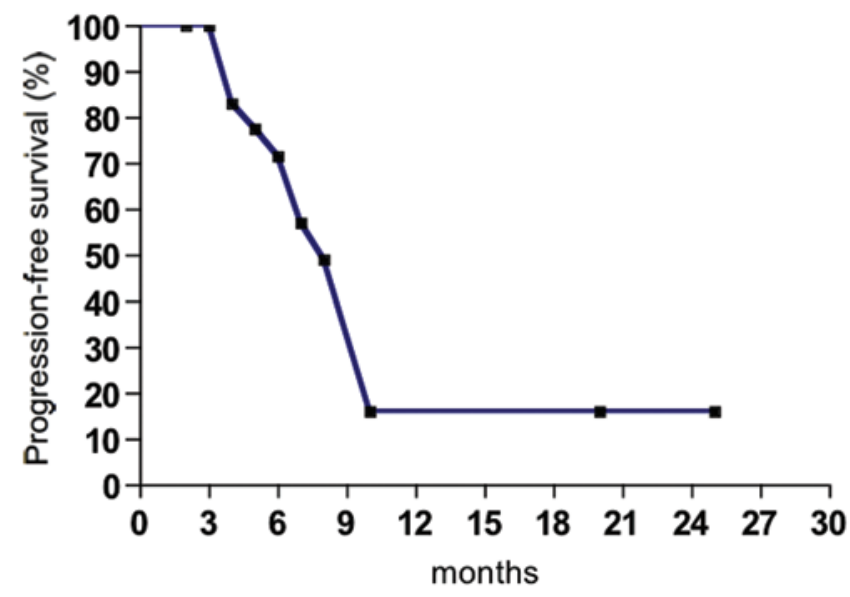

FIG. 5. Kaplan-Meier curve of progression-free survival. could lead to a reduced accuracy with respect to tumor identification. ${ }^{29}$ Nevertheless, different studies performed with and without filters on the surgical microscope have shown a high level of accuracy in tumor identification. ${ }^{8}$ These data were confirmed by our analysis of biopsies of fluorescent and nonfluorescent tissue at the tumor margin, with a sensitivity and specificity in tumor identification of $94 \%$ and $89.5 \%$, respectively. The use of a specific filter on the surgical microscope could have led to better accuracy in tumor identification. This could also explain why the patients did not experience a statistically significant difference in neurological function in the postoperative period. In addition, the new filter, YELLOW 560, allowed a dose reduction of fluorescein, due to the higher specificity of the filter characteristics. Theoretically, this could further reduce the number of side effects. Furthermore, this filter allowed an optimal delineation of fluorescent tissue, together with a good visualization of the peritumoral area in more natural colors, thus allowing the surgeon to complete most of the procedure without the need to switch the microscope illumination to normal white light, as happens during 5-ALA-guided resection (Figs. 1 and 2).

The median duration of long-term follow-up (10 months) is still not sufficient to draw a definitive conclusion about long-term outcome. However, preliminary data

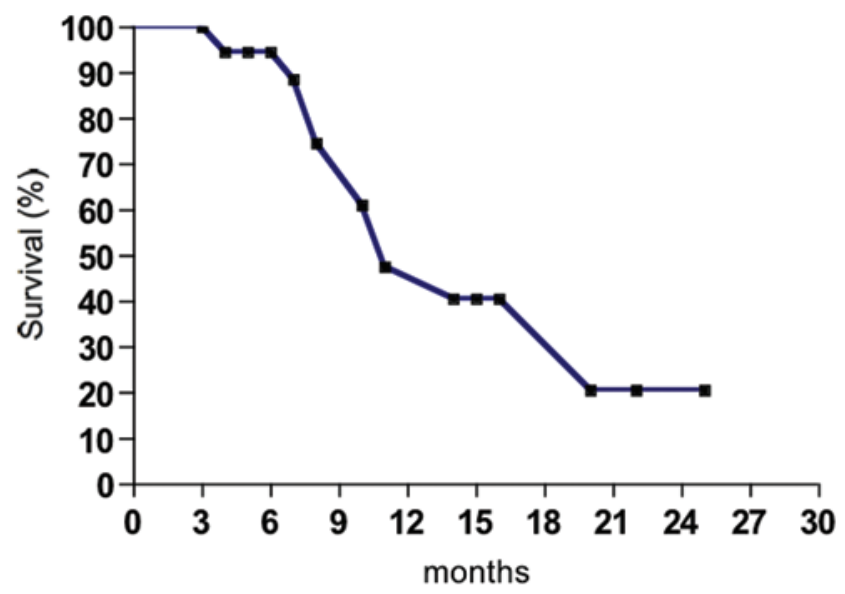

Fig. 6. Kaplan-Meier curve of overall survival. 
on 6-month PFS were promising, with more than $70 \%$ of patients showing no disease progression.

\section{Conclusions}

Based on the data collected in these 20 patients, the use of intravenous fluorescein during surgery for HGGs with a dedicated filter integrated into the surgical microscope appears safe and able to allow a high rate of complete resection. If confirmed at the end of the study, these results will represent the prerequisite to build up a proper prospective randomized controlled Phase III trial to definitively prove a positive effect of intravenous fluorescein on extent of resection of HGGs.

\section{Acknowledgments}

We would like to thank Dr. Cristina Montomoli for her help in statistical analysis, and Dr. Raffaele Nunziata for his help in histological analysis.

\section{Disclosure}

The authors report no conflict of interest concerning the materials or methods used in this study and the findings specified in the paper.

Author contributions to the study and manuscript preparation include the following. Conception and design: Acerbi, M Broggi. Acquisition of data: Acerbi, M Broggi, Eoli, Anghileri, Cavallo, Boffano, Cordella, Cuppini, Pollo, Schiariti, Visintini, La Corte. Analysis and interpretation of data: Acerbi, M Broggi, Eoli, Anghileri, Cavallo, Ferroli. Drafting the article: Acerbi. Critically revising the article: Acerbi, G Broggi, Ferroli. Statistical analysis: Orsi. Study supervision: G Broggi, Ferroli.

\section{References}

1. Acerbi F, Broggi M, Eoli M, Anghileri E, Cuppini L, Pollo B, et al: Fluorescein-guided surgery for grade IV gliomas with a dedicated filter on the surgical microscope: preliminary results in 12 cases. Acta Neurochir (Wien) 155:1277-1286, 2013

2. Cordella R, Acerbi F, Broggi M, Vailati D, Nazzi V, Schiariti $\mathrm{M}$, et al: Intraoperative neurophysiological monitoring of the cortico-spinal tract in image-guided mini-invasive neurosurgery. Clin Neurophysiol 124:1244-1254, 2013

3. Dilek O, Ihsan A, Tulay H: Anaphylactic reaction after fluorescein sodium administration during intracranial surgery. J Clin Neurosci 18:430-431, 2011

4. Eoli M, Menghi F, Bruzzone MG, De Simone T, Valletta L, Pollo B, et al: Methylation of O6-methylguanine DNA methyltransferase and loss of heterozygosity on 19q and/or 17p are overlapping features of secondary glioblastomas with prolonged survival. Clin Cancer Res 13:2606-2613, 2007

5. Koc K, Anik I, Cabuk B, Ceylan S: Fluorescein sodium-guided surgery in glioblastoma multiforme: a prospective evaluation. Br J Neurosurg 22:99-103, 2008

6. Kubben PL, ter Meulen KJ, Schijns OE, ter Laak-Poort MP, van Overbeeke JJ, van Santbrink H: Intraoperative MRI-guided resection of glioblastoma multiforme: a systematic review. Lancet Oncol 12:1062-1070, 2011

7. Kuhnt D, Becker A, Ganslandt O, Bauer M, Buchfelder M, Nimsky C: Correlation of the extent of tumor volume resection and patient survival in surgery of glioblastoma multiforme with high-field intraoperative MRI guidance. Neuro Oncol 13:1339-1348, 2011

8. Kuroiwa T, Kajimoto Y, Ohta T: Development of a fluorescein operative microscope for use during malignant glioma surgery: a technical note and preliminary report. Surg Neurol 50:41-49, 1998

9. Kwan AS, Barry C, McAllister IL, Constable I: Fluorescein angiography and adverse drug reactions revisited: the Lions Eye experience. Clin Experiment Ophthalmol 34:33-38, 2006

10. Kwiterovich KA, Maguire MG, Murphy RP, Schachat AP, Bressler NM, Bressler SB, et al: Frequency of adverse systemic reactions after fluorescein angiography. Results of a prospective study. Ophthalmology 98:1139-1142, 1991

11. Lacroix M, Abi-Said D, Fourney DR, Gokaslan ZL, Shi W, DeMonte F, et al: A multivariate analysis of 416 patients with glioblastoma multiforme: prognosis, extent of resection, and survival. J Neurosurg 95:190-198, 2001

12. Louis DN, Ohgaki H, Wiestler OD, Cavenee WK: WHO Classification of Tumours of the Central Nervous System. Lyon: IARC Press, 2007

13. McGirt MJ, Chaichana KL, Gathinji M, Attenello FJ, Than K, Olivi A, et al: Independent association of extent of resection with survival in patients with malignant brain astrocytoma. Clinical article. J Neurosurg 110:156-162, 2009

14. Moore GE, Peyton WT, French LA, Walker WW: The clinical use of fluorescein in neurosurgery. The localization of brain tumors. J Neurosurg 5:392-398, 1948

15. Nimsky C, Ganslandt O, Buchfelder M, Fahlbusch R: Intraoperative visualization for resection of gliomas: the role of functional neuronavigation and intraoperative 1.5 T MRI. Neurol Res 28:482-487, 2006

16. Novotny HR, Alvis DL: A method of photographing fluorescence in circulating blood in the human retina. Circulation 24:82-86, 1961

17. Pollo B: Neuropathological diagnosis of brain tumours. Neurol Sci 32 (Suppl 2):S209-S211, 2011

18. Schebesch KM, Proescholdt M, Höhne J, Hohenberger C, Hansen E, Riemenschneider MJ, et al: Sodium fluorescein-guided resection under the YELLOW $560 \mathrm{~nm}$ surgical microscope filter in malignant brain tumor surgery-a feasibility study. Acta Neurochir (Wien) 155:693-699, 2013

19. Senft C, Bink A, Franz K, Vatter H, Gasser T, Seifert V: Intraoperative MRI guidance and extent of resection in glioma surgery: a randomised, controlled trial. Lancet Oncol 12:9971003, 2011

20. Shinoda J, Yano H, Yoshimura S, Okumura A, Kaku Y, Iwama $\mathrm{T}$, et al: Fluorescence-guided resection of glioblastoma multiforme by using high-dose fluorescein sodium. Technical note. J Neurosurg 99:597-603, 2003

21. Stummer W, Meinel T, Ewelt C, Martus P, Jakobs O, Felsberg J, et al: Prospective cohort study of radiotherapy with concomitant and adjuvant temozolomide chemotherapy for glioblastoma patients with no or minimal residual enhancing tumor load after surgery. J Neurooncol 108:89-97, 2012

22. Stummer W, Novotny A, Stepp H, Goetz C, Bise K, Reulen HJ: Fluorescence-guided resection of glioblastoma multiforme by using 5-aminolevulinic acid-induced porphyrins: a prospective study in 52 consecutive patients. J Neurosurg 93:10031013,2000

23. Stummer W, Pichlmeier U, Meinel T, Wiestler OD, Zanella F, Reulen HJ: Fluorescence-guided surgery with 5-aminolevulinic acid for resection of malignant glioma: a randomised controlled multicentre phase III trial. Lancet Oncol 7:392-401, 2006

24. Stummer W, Stocker S, Novotny A, Heimann A, Sauer O, Kempski O, et al: In vitro and in vivo porphyrin accumulation by C6 glioma cells after exposure to 5-aminolevulinic acid. J Photochem Photobiol B 45:160-169, 1998

25. Stummer W, Stocker S, Wagner S, Stepp H, Fritsch C, Goetz C, et al: Intraoperative detection of malignant gliomas by 5 -aminolevulinic acid-induced porphyrin fluorescence. Neurosurgery 42:518-526, 1998 


\section{F. Acerbi et al.}

26. Stupp R, Hegi ME, Mason WP, van den Bent MJ, Taphoorn MJ, Janzer RC, et al: Effects of radiotherapy with concomitant and adjuvant temozolomide versus radiotherapy alone on survival in glioblastoma in a randomised phase III study: 5-year analysis of the EORTC-NCIC trial. Lancet Oncol 10:459466, 2009

27. Stupp R, Mason WP, van den Bent MJ, Weller M, Fisher B, Taphoorn MJ, et al: Radiotherapy plus concomitant and adjuvant temozolomide for glioblastoma. N Engl J Med 352:987-996, 2005

28. Tanahashi S, Lida H, Dohi S: An anaphylactoid reaction after administration of fluorescein sodium during neurosurgery. Anesth Analg 103:503-504, 2006 (Letter)

29. Tonn JC, Stummer W: Fluorescence-guided resection of malignant gliomas using 5-aminolevulinic acid: practical use, risks, and pitfalls. Clin Neurosurg 55:20-26, 2008

30. Unsgaard G, Ommedal S, Muller T, Gronningsaeter A, Nagelhus Hernes TA: Neuronavigation by intraoperative three-dimensional ultrasound: initial experience during brain tumor resection. Neurosurgery 50:804-812, 2002

31. Wen PY, Macdonald DR, Reardon DA, Cloughesy TF, Sorensen AG, Galanis E, et al: Updated response assessment criteria for high-grade gliomas: response assessment in neurooncology working group. J Clin Oncol 28:1963-1972, 2010

32. Wirtz CR, Albert FK, Schwaderer M, Heuer C, Staubert A, Tronnier VM, et al: The benefit of neuronavigation for neurosurgery analyzed by its impact on glioblastoma surgery. Neurol Res 22:354-360, 2000

33. Yannuzzi LA, Rohrer KT, Tindel LJ, Sobel RS, Costanza MA, Shields W, et al: Fluorescein angiography complication survey. Ophthalmology 93:611-617, 1986

Manuscript submitted October 15, 2013.

Accepted November 21, 2013.

Portions of this paper were presented in abstract form at the XV WFNS World Congress of Neurosurgery, Seoul, Korea, September 8-13, 2013.

Please include this information when citing this paper: DOI: 10.3171/2013.11.FOCUS13487.

Address correspondence to: Francesco Acerbi, M.D., Ph.D., Fondazione IRCCS Istituto Neurologico Carlo Besta, Via Celoria 11, Milano 20133, Italy.email: acerbi.f@istituto-besta.it. 